\title{
Selected topics from the 25th International Colloquium on the Dynamics of Explosions and Reactive Systems, Leeds, UK, August 2-7, 2015
}

\author{
G. Ciccarelli ${ }^{1}$
}

Published online: 11 August 2016

(C) Springer-Verlag Berlin Heidelberg 2016

This issue contains selected papers that were presented at the 25th International Colloquium on the Dynamics of Explosions and Reactive Systems (ICDERS) held in Leeds, UK, on July 28-August 2, 2015. The biennial ICDERS is the reference meeting focusing specifically on dynamic aspects of reactive systems. The local organization was headed by Malcom Lawes (University of Leeds). The program committee was chaired by Matei Radulescu (University of Ottawa) and co-chaired by Aslan Kasimov, Akiko Matsuo, and Antonio Sanchez, with the assistance of Benjamin Akih Kumgeh, Nabiha Chaumeix, Ashwin Chinnayya, Sergey Frolov, Scott I. Jackson, Jiro Kasahara, Eric Petersen, and Alexei Poludnenko.

A total of 327 participants attended the conference, of which 211 delivered oral presentations and 63 presented posters on explosions, detonations, ignition, turbulent combustion, and other topics in combustion. With 22 countries represented, the conference was truly international. As in previous years, presenters at the conference were encouraged to submit their work to a selection of three journals depending on the research topic. The current issue includes papers dealing with combustion in high-speed flow and shock waves, detonation, and deflagration-to-detonation transition, whose authors elected to submit to Shock Waves. Submissions were subjected to the same rigorous review process as a regular submission to Shock Waves. Each paper was reviewed by at least two independent referees, each of whom is an internationally recognized expert in the field.

G. Ciccarelli

ciccarel@me.queensu.ca

1 Department of Mechanical and Materials Engineering, Queen's University, 130 Stuart Street, McLaughlin Hall, Kingston, ON K7L 3N6, Canada
Nine papers from the conference were submitted, of which one was withdrawn by the authors after an initial "major revision" decision. The remaining eight papers were eventually accepted, upon two or more revisions, after a consensus was reached by the referees and the editor. Two of the submissions were published as Technical Notes due to their limited scope.

The current issue includes papers on the kinetics of hydrogen combustion, triple-shock reflection accounting for viscous effects, detonation in ethylene-air-water spray, and three papers involving DDT in a smooth and obstacle-filled channel. Six additional papers in the issue did not originate from the conference but are included due to their high relevance to the topics covered at ICDERS. Geographically, papers from the conference originated from Canada, China, France, Germany, Japan, and the USA.

I wish to thank the authors who submitted their papers to this issue and then revised their papers numerous times to produce the best quality possible. I would also like to thank the many reviewers whose help was crucial in ensuring the high quality of the papers that appear in this issue. I would like to thank both the authors and reviewers for their quick responses making it possible to publish the ICDERS issue online within one year of the conference date. 